\title{
Evaluation of a multi-sensor horizontal dual arm Coordinate Measuring Machine for automotive dimensional inspection
}

\author{
Glen A. Turley • Ercihan Kiraci • Alan Olifent • \\ Alex Attridge • Manoj K. Tiwari • Mark A. Williams
}

Received: 8 November 2013 / Accepted: 23 February 2014 / Published online: 30 March 2014

(c) The Author(s) 2014. This article is published with open access at Springerlink.com

\begin{abstract}
Multi-sensor coordinate measuring machines (CMM) have a potential performance advantage over existing CMM systems by offering the accuracy of a touch trigger probe with the speed of a laser scanner. Before these systems can be used, it is important that both random and systematic errors are evaluated within the context of its intended application. At present, the performance of a multisensor CMM, particularly of the laser scanner, has not been evaluated within an automotive environment. This study used a full-scale CNC machined physical representation of a sheet metal vehicle body to evaluate the measurement agreement and repeatability of critical surface points using a multi-sensor horizontal dual arm CMM. It was found that there were errors between CMM arms and with regard to part coordinate frame construction when using the different probing systems. However, the most significant effect upon measurement error was the spatial location of the surface feature. Therefore, for each feature on an automotive assembly, measurement agreement and repeatability has to be individually determined to access its acceptability for measurement with a laser scanner to improve CMM utilisation, or whether the accuracy of a touch trigger probe is required.
\end{abstract}

G. A. Turley $(\bowtie) \cdot$ E. Kiraci · A. Attridge $\cdot$ M. A. Williams WMG, The University of Warwick, Coventry, CV4 7AL, UK e-mail: glen.turley@warwick.ac.uk

\section{A. Olifent}

Jaguar Land Rover Limited, Abbey Road, Whitley, Coventry CV3

4LF, UK

\section{K. Tiwari}

Indian Institute of Technology Kharagpur, Kharagpur, 721302, India
Keywords CMM · Laser scanner · Measurement systems assessment

\section{Introduction}

Accurate measurements are important in being able to monitor production processes and ensure conformity to design specifications [1]. In the automotive industry, inspection of key dimensional and geometrical tolerances is typically done using a coordinate measuring machine (CMM) $[2,3]$. Traditionally, a touch trigger probe has been employed as the CMM sensing mechanism because of well-established calibration processes and knowledge of measurement uncertainties $[4,5]$. More recently, due to the increase in accuracy of non-contact laser triangulation sensors (LTS), CMM machines with both contact and non-contact sensing systems are becoming common $[1,5]$. LTS digitising, more commonly referred to as laser scanning, offer advantages over the touch trigger method with regards to faster measurement speed, higher resolution and non-contact measurement to prevent local part deformation during inspection [6-8]. All three are important for automotive manufacturers offering improvements in CMM utilisation, better estimation of feature characteristics as well as measurement validity by preventing sheet metal and plastic component displacement during inspection [2,9]. CMM utilisation is an area of particular interest, as greater measurement throughput will provide a better estimation of process capability and prevent the need for further CMM investment. However, while laser scanning is well-established in the reverse engineering field and in the inspection of freeform surfaces, lack of knowledge about measurement uncertainties has meant their use in the dimensional control of mechanical parts has been more limited $[4,10,11]$. This is particularly 
true with regard to large volume components, such as automotive assemblies, where measurement accuracy depends upon both the surface material and the geometrical elements which have to be measured [11]. Both of these factors affect laser scanner measurement uncertainty as the technology is sensitive to issues such as colour, surface roughness and reflectivity which do not influence tactile measurements $[4,12]$. However, it has been recognised that the greater point density provided by laser scanners can lead to better conditioned fitting algorithms for characteristics such as the diameter or centre point of a sphere or circle [7, 13]. Multi-sensor data fusion, which combines data from two or more sensors in a common spatial representational format, has been proposed as a way to provide greater measurement information while maintaining or improving the measurement uncertainty [11]. Consequently, the combination of using a touch trigger probe in conjunction with a laser scanner has the potential to provide accuracy, speed as well as detailed surface information about the measured artefact. However, because the measurement uncertainty of a laser scanner is affected by both the artefact being scanned and the particular surface feature, an evaluation of a multisensor CMM needs to be relevant to its intended purpose. Therefore, the main objective of this study was to use an automotive artefact to assess the suitability of laser scanning in the dimensional inspection of an automotive vehicle body as part of a multi-sensor solution.

\section{Background}

A CMM is a computer numerically controlled (CNC) measurement system used to detect the spatial coordinates of an artefact surface with the aid of a probing device. It typically has 5 degrees of freedom with three X,Y,Z translations and a further two 'A' and ' $\mathrm{B}$ ' rotations at the probe head. The $\mathrm{X}, \mathrm{Y}, \mathrm{Z}$ translations provide controlled displacements within the work envelope of the CMM while the 'A' and ' $\mathrm{B}$ ' rotations ideally orientate the measurement probe with respect to the artefact surface $[7,12]$. The touch trigger probe is a tactile sensing element which uses nominal (theoretically perfect) part geometry to approach normal to the artefact surface and triggers once contact has been made. The CMM treatment system corrects this measured point according to known error variables derived from the CMM calibration to provide a comparison with the nominal part geometry [14]. In contrast, the laser scanning probe consists of a transmitter that emits a focussed laser line onto the artefact surface which diffuses the laser light. This diffuse reflection is filtered by the receiving lens of the laser scanner and focussed to form a 2D laser image on the LTS photo detector $[6,15]$. The CMM calibration then provides an error-compensated transformation which converts the coordinates of the photo detector 2D image into the 3D spatial coordinates of the CMM [15]. Therefore, as the laser scanner moves across the artefact surface, detailed 3D point cloud information is generated which can be compared with the nominal part geometry. The different data acquisition methods of the touch trigger and laser scanning probes cause systematic errors which affect the agreement between the measurement results and also random errors which affect individual probe repeatability.

\subsection{Laser scanner measurement uncertainties}

There have been a number of published tests which have evaluated the measurement agreement and repeatability of CMM-mounted laser scanning probes compared to touch trigger probes. These experiments used a variety of different artefacts which included stand-alone reference plates [12, 16-19], reference plates combined with reference spheres $[6,7]$, reference cylinders [4] and truncated pyramids [7]. There have also been artefacts containing a range of different geometries such as holes, slots, fillets and chamfers $[2,5]$. The findings of these studies have uncovered many extrinsic parameters which can affect the measurement uncertainty of laser scanners which have been summarised below, although recent advances have sought to minimise these effects.

Scan depth Each laser scanning probe has a specified field of view, as shown in Fig. 1. This field of view represents the window in which the laser scanner photo detector can acquire measurement points from the scanned surface [12]. When the laser scanner is positioned such that the artefact surface is located at the beginning of this field of view, the acquired point cloud will have a higher resolution and lower random error compared to if it was located at the end of the field of view. Studies have also found a systematic error of $15 \mu \mathrm{m}$ between scans taken at the beginning of the field of view compared to the end when the laser scanner is further away from the artefact surface $[16,17]$. When scanning outside of the field of view, the performance of the laser scanning probe deteriorates significantly [20].

Incident angle The in-plane $(\alpha)$ and out-of-plane $(\beta)$ scanning angles, shown in Fig. 1, can affect the quality of the acquired point cloud [7]. Laser intensity is at its maximum when the in-plane and out-of-plane angles are perpendicular to the surface; this can cause point saturation resulting in positioning errors [12]. A further benefit of not scanning perpendicular to the surface is that random error is distributed more evenly between the individual $x$-, $y$ - and $z$-axes. This is due to measurement noise principally being affected by the scan depth which acts uni-directionally [17]. However, there is a limit with regard to how far away from 


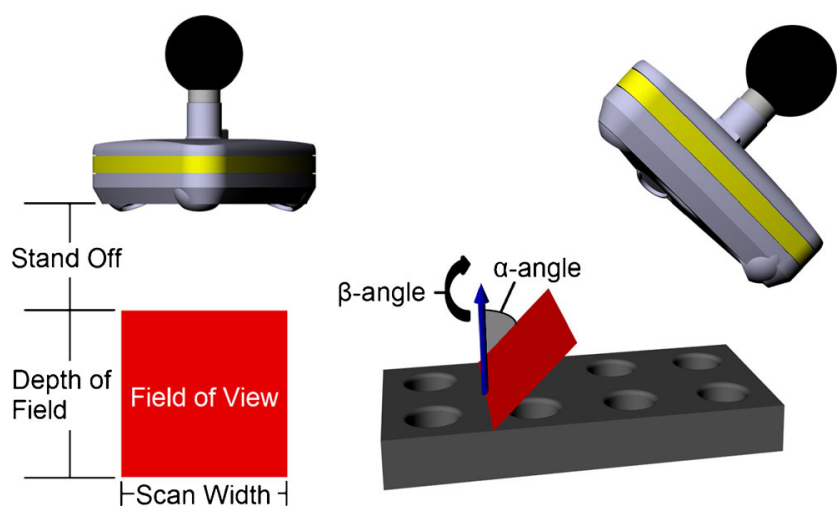

Fig. 1 Illustration of laser scanning parameters: Stand-off, field of view (scan depth and width), in-plane angle $(\alpha)$, and out-of-plane angle $(\beta)$

perpendicular the laser scanner can be compared to the artefact surface. Once the incident angle goes beyond $60^{\circ}$, the intensity of the reflected laser light is too small to be detected [16].

Probe head orientation The laser scanning probe can be ideally orientated to the artefact surface by changing the ' $A$ ' and ' $B$ ' $C M M$ rotational axes. However, using multiple probe angles increases the time it takes to measure the artefact as well as the time to qualify a greater amount of probe angles, without improving accuracy $[2,5]$. Other investigations have also shown that there is a lack of agreement between point clouds obtained using different probe head orientations $[4,10]$. However, multiple probe angles cannot be avoided as they are necessary when scanning small objects in order to measure enough of the artefact surface to be able to have a well-conditioned fitting algorithm $[5,17]$.

Surface properties Both colour and reflectivity of the scanned surface influence the measurement result. Dark surfaces or colours reflect the minimal intensity of light making point acquisition difficult $[2,21]$. On the other hand, smooth metallic materials are not reflective within the range of the laser light spectrum which again makes point acquisition difficult $[12,16]$. Ideally, diffuse reflection is preferred as it reflects equally in all directions maintaining scan quality when measuring from different angles [16].

These findings have led to improved measurement practices and with the laser scanning technology itself, particularly with regard to how it handles different surface properties [5, 17]. However, they reveal that measurement uncertainties are dependent upon the intended application. Therefore, a measurement study needs to be designed that is relevant to the automotive industry.

\subsection{Study objectives}

There are a number of characteristics which distinguish the requirements for an automotive measurement task from the previous evaluation studies. Firstly, measurement of a vehicle body shell and completed vehicles is generally done with a horizontal dual arm CMM, as shown in Fig. 2. Secondly, vehicle geometry is generally complex with a number of occlusions. Therefore, different probe angles are required to measure surface features, sometimes with a less than ideal incident angle. The features that are required to be evaluated are discrete surface points, holes and slots rather than free form geometry. Finally, the global coordinate frame of the CMM is required to be transformed to the local part coordinate frame of the vehicle through the measurement of defined datum features. All these factors have the potential to affect measurement uncertainty. The current method for the verification of a CMM is controlled by ISO 10360-2 whereby its measurement uncertainty and repeatability is determined through the measurement of a calibrated test length which has direct traceability to the metre unit [22]. Therefore, the purpose of this study was to evaluate a multi-sensor CMM whose performance had been verified to international standards for its suitability for a complex automotive inspection task. The specific study objectives were (1) to evaluate the random and systematic errors of a horizontal dual arm CMM using a multi-sensor probing system and (2) to assess the impact of using a multi-sensor approach upon the dimensional inspection of automotive assemblies.

\section{Materials and methods}

To evaluate the capability of a multi-sensor probing system, a LK H Horizontal Dual Arm CMM was utilised (Nikon Metrology, UK) - pictured in Fig. 2. The configuration used is seen regularly in the automotive industry and provides

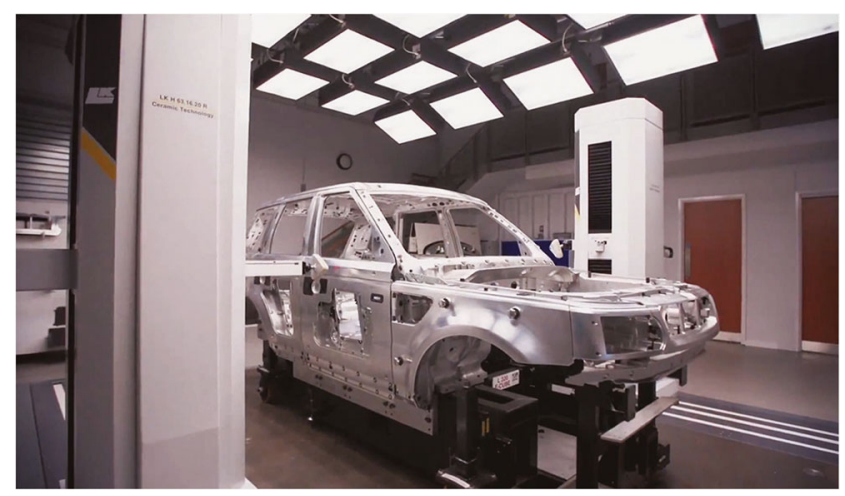

Fig. 2 Horizontal dual arm CMM with Land Rover Environmental Cube (E-Cube) CNC machined vehicle body simulator located on measurement bed 
measurement access to the exterior, interior and underbody of the vehicle. Mounted on each of the CMM horizontal measuring arms was a PH10MQ indexing probe head (Renishaw, UK) which was used in conjunction with an ACR3 probe changing rack (Renishaw, UK) to allow automatic changing between touch trigger and laser scanning probes within the measurement program. For contact measurement, a TP20 5-way kinematic standard force touch trigger probe was used with $140-\mathrm{mm}$ extension and 20-mm long by $2-\mathrm{mm}$ diameter stylus (Renishaw, UK). For non-contact measurement, a Metris XC65Dx (Nikon Metrology, Belgium) laser scanner was used, shown in Fig. 1. This laser scanner emits three laser lines in a cross hatch formation each having a scan depth of $65 \mathrm{~mm}$, scan width of $65 \mathrm{~mm}$ and a standoff distance of $75 \mathrm{~mm}$ allowing in total 75,000 points per second to be captured. Prior to the measurement experiment, both CMM arms when fitted with a touch trigger probe were verified in accordance with ISO10360-2 and had an expanded measurement uncertainty $(k=2)$ of within $\pm 1.0 \mu \mathrm{m}+1.0 \mu \mathrm{m} / \mathrm{m}$. The Metris XC65Dx laser scanners were verified to the same standard achieving a measurement uncertainty of within $12 \mu \mathrm{m}$ when measuring a ceramic sphere [22]. To evaluate the performance of the certified multi-sensor horizontal dual arm CMM for a complex automotive measurement task, an aluminium CNC machined full-scale physical representation of the manufactured sheet metal body of a vehicle (Jaguar Land Rover Limited, UK) was selected. This artefact is known as an environmental cube (E-Cube) and is shown in Fig. 2 [23]. The E-Cube has all the interior and exterior surface features of a vehicle body to allow fitment of trim components for quality maturation purposes to assess their fit, finish and alignment during product development [24, 25]. The following sections detail the artefact experimental set-up, the measurement study and statistical analysis procedures for this study.

\subsection{Experimental set-up}

The E-Cube had machined flat faces on its base to enable it to locate on the CMM measurement bed without the need of a separate measurement fixture. The E-Cube remained on the measurement bed for the duration of the study and the environment was maintained at a standard $20^{\circ} \mathrm{C} \pm 1^{\circ} \mathrm{C}$ throughout [26]. To transform the global coordinate frame of the CMM into the local part coordinate frame of the vehicle, four datum plates located at the front and rear lower corners of the E-Cube were measured-two with the LH horizontal CMM arm and two with the RH CMM arm. Figure 3 provides a schematic of the local coordinate frame. To construct the local $x$-axis, the mid-point between the two front datum plates and between the two rear datum plates were determined and a line constructed between them; the unit vector of this line formed the $x$-axis according to Eq. 1 .

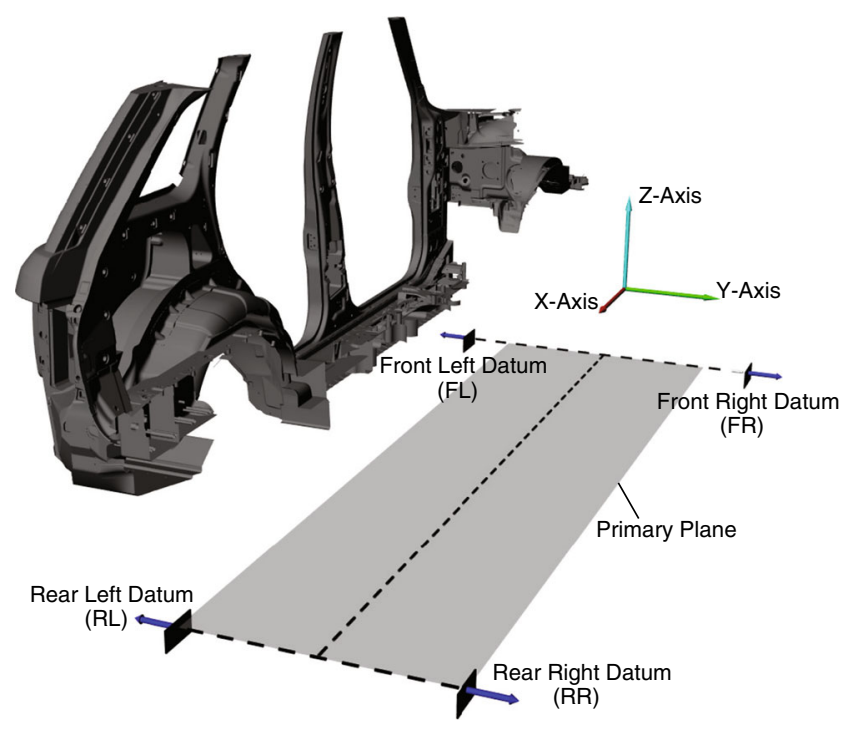

Fig. 3 Schematic of the E-Cube local part coordinate frame using the front and rear datum plates

The local $z$-axis was constructed normal to a plane formed between the mid-point of the two front datum plates and both of the rear datum plates. To do this, the vector crossproduct was calculated using the $x$-axis vector and a vector formed by the line between the two rear datum plates, (2). The vector cross-product between the $x$ - and $z$-axes was then used to form the $y$-axis, (3). The $x$-, $y$ - and $z$-axes unit vectors were then combined to form the transformation matrix $\mathbf{R}$, (4). This matrix $\mathbf{R}$ was calculated using both the CMM measured values $\left(\mathbf{R}_{C M M}\right)$ as well as using the nominal values of the E-Cube datum plates $\left(\mathbf{R}_{C u b e}\right)$. Any measured value in the CMM global coordinate frame could then be transformed into the local part coordinate frame using Eq. 5. Finally, the rotated local coordinate frame was translated so that its origin coincided with the nominal origin of the local part coordinate frame, completing the reference point system alignment (RPS).

$$
\begin{aligned}
& \mathbf{x}_{\text {axis }}=\frac{\frac{1}{2}\left(F_{L}+F_{R}\right)-\frac{1}{2}\left(F_{L}+F_{R}\right)}{\left\|\frac{1}{2}\left(F_{L}+F_{R}\right)-\frac{1}{2}\left(F_{L}+F_{R}\right)\right\|} \\
& \mathbf{z}_{\text {axis }}=\mathbf{x}_{\text {axis }} \times \frac{R_{L}-R_{R}}{\left\|R_{L}-R_{R}\right\|} \\
& \mathbf{y}_{\text {axis }}=\mathbf{x}_{\text {axis }} \times \mathbf{z}_{\text {axis }}
\end{aligned}
$$

$\mathbf{R}=\left[\begin{array}{lll}x_{1} & x_{2} & x_{3} \\ y_{1} & y_{2} & y_{3} \\ z_{1} & z_{2} & z_{3}\end{array}\right]$

$$
\mathbf{R}_{\text {carline }}=\mathbf{R}_{\text {Cube }}^{T} \cdot \mathbf{R}_{C M M}
$$




\subsection{Experimental procedure}

To evaluate the systematic error between the two horizontal CMM arms, a ceramic reference sphere (Kolb + Baumann, Germany) of $29.9912 \mathrm{~mm}$ diameter was measured by the touch trigger probe mounted on each of the horizontal CMM arms. This sphere was measured within the CMM global coordinate frame. This procedure was repeated for the laser scanning probe later in the measurement program. Following measurement of the reference sphere, the E-Cube datum plates were measured with the touch trigger probe to construct the local coordinate frame detailed in Section 3.1. This process was iterated twice to improve the positional accuracy of the non-critical axes of the E-Cube datum plates in comparison to their nominal values. The established local coordinate frame was defined as $\mathbf{R}_{\text {Touch }}$ and was referred to as such throughout the duration of the study. After establishing the local coordinate frame $\mathbf{R}_{\text {Touch }}$, a total of 18 surface points were measured by the horizontal dual arm CMM. The nine surface points measured by the RH CMM arm were a mirror image of the surface points measured by the $\mathrm{LH}$ CMM arm-three in each of the $x$-, $y$ - and $z$-axes, as shown in Fig. 4. These measurement points represent a sub-set of the critical surface points required to be measured to assess the fitment of both interior and exterior components such as doors, interior headlining and rear bumper. Surface points were also selected to ensure that errors were evaluated in each measurement axis throughout the working envelope of the vehicle. On completion, the touch trigger probe was changed for the Metris XC65Dx laser scanner via the ACR3 probe changing rack.

To evaluate the systematic error in comparison to the touch trigger probe, the E-Cube surface points were measured again using the same $\mathbf{R}_{\text {Touch }}$ local coordinate frame. Direct comparison of the touch trigger probe measurements was achieved by constructing a localised triangulated mesh from the laser scanner point cloud data. From this constructed surface, the $\mathrm{x}, \mathrm{y}, \mathrm{z}$ nominal and $\mathrm{i}, \mathrm{j}, \mathrm{k}$ vector information was used to extract each individual surface point feature. The surface points were measured using a 'sheetmetal' profile having maximum laser intensity with a long exposure. A point spacing of $0.1 \mathrm{~mm}$ along and between the laser lines was also set. Once complete, the CMM global coordinate frame was reloaded and the E-Cube datum plates were re-measured with the laser scanning probe to establish a second local coordinate frame, referred to as $\mathbf{R}_{\text {Laser }}$. This was to evaluate the systematic error that occurs when constructing a local part coordinate frame using two different probing technologies. Measurement of the E-Cube datum plates was iterated twice, in the same manner as the touch trigger probe. The datum plates were measured using a 'dark matt' profile with maximum laser intensity but slightly shorter exposure compared to the 'sheet metal' profile. The same E-Cube surface points were measured for a final time with the laser scanner with the $\mathbf{R}_{\text {Laser }}$ local coordinate frame. The measurement routine was repeated 30 times to assess measurement system random error. The experiment took place with the absence of light, except for three low-intensity emergency lights located on the LH wall of the laboratory.

\subsection{Statistical analysis}

To compare the systematic error between the two CMM horizontal arms, the mean position and diameter values of the ceramic reference sphere were calculated from the 30 sets of measurements. In total, there were two comparisons, one for when the LH and RH CMM arms were fitted with the touch trigger probe and a second when the CMM arms were fitted with the laser scanning probe. Random error, or repeatability, was also evaluated using the calculated $2 \sigma$ standard deviation values of the 30 ceramic reference sphere measurements. Similarly, as an initial analysis of the random and systematic error present between the touch trigger probe and
Fig. 4 Illustration of the E-Cube surface point locations measured during experiment (left-hand side only)

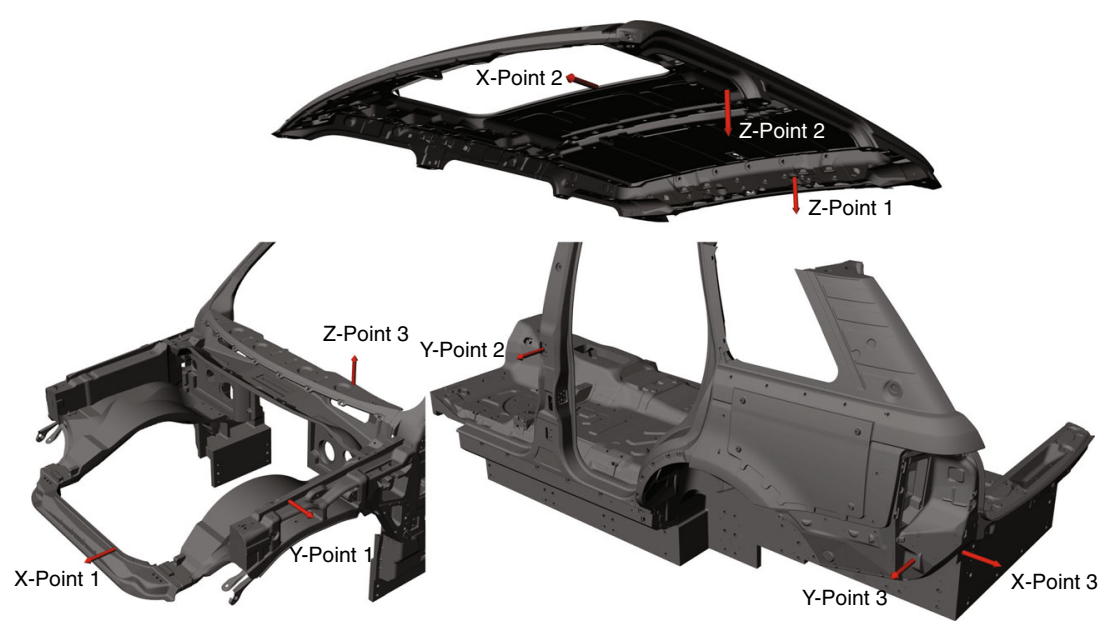


the laser scanner, the mean error and $2 \sigma$ repeatability values of the 18 E-Cube surface point measurements were calculated and compared. This comparison was done for both sets of laser scanning measurements, once using the $\mathbf{R}_{\text {Touch }}$ local coordinate frame and a second when the E-Cube surface points were measured using the $\mathbf{R}_{\text {Laser }}$ coordinate frame. To evaluate the error between the $\mathbf{R}_{\text {Touch }}$ and $\mathbf{R}_{\text {Laser }}$ local part coordinate frames, the deviation in the $x$-, $y$ - and $z$-axes between the origin of the $\mathbf{R}_{\text {Touch }}$ coordinate frame and the origin of $\mathbf{R}_{\text {Laser }}$ coordinate frame was calculated for all 30 sets of measurements. As well as the translational difference between the coordinate frame origins, the angle between the axes of the two local coordinate frames was also calculated. To evaluate the impact of coordinate frame error upon the E-Cube surface point measurements, the positional error for each surface point between the reference $\mathbf{R}_{\text {Touch }}$ coordinate frame and the $\mathbf{R}_{\text {Laser }}$ coordinate frame was calculated. To do this, for each of the 30 sets of measurements, the nominal $\mathrm{x}, \mathrm{y}, \mathrm{z}$ coordinates of the $18 \mathrm{E}$-Cube surface points were transformed from the reference $\mathbf{R}_{\text {Touch }}$ coordinate frame into the $\mathbf{R}_{\text {Laser }}$ coordinate frame using Eq. 5.

To evaluate the overall measurement agreement between the laser scanner measurements of the E-Cube surface points using $\mathbf{R}_{\text {Touch }}$ and $\mathbf{R}_{\text {Laser }}$ local coordinate frames, a crossed two-factor analysis of variance (ANOVA) was performed. To remove the effect of part error from this analysis, the mean of the 30 sets of touch-trigger probe measurements were deducted from both sets of laser scanner measurements. Therefore, any measurement deviation was directly related to a systematic error with the reference touch trigger probe. The ANOVA test evaluated the effect that (a) part coordinate frame and (b) location of the measured surface point had upon the agreement between measurement results, along with their combined effect. Effect size was calculated for any significant variable $(p<0.05)$ using omega-squared $\left(\omega^{2}\right)$ with the values $0.01,0.06$ and 0.14 , representing a small, medium and large effect [27]. All calculations were performed in SPSS version 21 (IBM Corp, Armonk, NY) with a significance level of 0.05 .

\section{Results}

The measurement of the ceramic reference sphere within the CMM global coordinate frame showed an error between the LH and RH horizontal CMM arms when fitted with the touch trigger probe of $10.9 \mu \mathrm{m}(2 \sigma: \mathrm{LH}=7.5 \mu \mathrm{m}$, $\mathrm{RH}=3.4 \mu \mathrm{m})$ in the $x$-axis, $1.4 \mu \mathrm{m}(2 \sigma: \mathrm{LH}=3.3 \mu \mathrm{m}$, $\mathrm{RH}=2.7 \mu \mathrm{m})$ in the $y$-axis and $11.0 \mu \mathrm{m}(2 \sigma: \mathrm{LH}=4.1 \mu \mathrm{m}$, $\mathrm{RH}=3.3 \mu \mathrm{m})$ in the $z$-axis. When measuring the same ceramic sphere with the laser scanner, the error was $3.1 \mu \mathrm{m}$ $(2 \sigma: \mathrm{LH}=28.9 \mu \mathrm{m}, \mathrm{RH}=29.5 \mu \mathrm{m})$ in the $x$-axis, $19.6 \mu \mathrm{m}$ $(2 \sigma: \mathrm{LH}=19.2 \mu \mathrm{m}, \mathrm{RH}=17.5 \mu \mathrm{m})$ in the $y$-axis and $13.1 \mu \mathrm{m}(2 \sigma: \mathrm{LH}=8.9 \mu \mathrm{m}, \mathrm{RH}=5.6 \mu \mathrm{m})$ in the $z-$ axis. The error compared to the calibrated diameter of the ceramic sphere was $9.6 \mu \mathrm{m}(2 \sigma=3.9 \mu \mathrm{m})$ with the $\mathrm{LH}$ CMM arm fitted with the touch trigger probe and $16.7 \mu \mathrm{m}$ $(2 \sigma=2.1 \mu \mathrm{m})$ with the RH CMM arm. When fitted with the laser scanner, the error was $27.1 \mu \mathrm{m}(2 \sigma=2.5 \mu \mathrm{m})$ with the LH CMM arm and $22.8 \mu \mathrm{m}(2 \sigma=1.5 \mu \mathrm{m})$ with the RH CMM arm.

For the E-Cube surface point measurements, Table 1 provides the median, minimum and maximum systematic error in each axis between the reference touch trigger probe and the laser scanner. This comparison includes laser scanner measurements using both part coordinate frames as well as the deviation between the two sets of laser scanner measurements. Overall, the deviation of the laser scanner away from the reference touch trigger probe was similar with both part coordinate frames: $\mathbf{R}_{\text {Touch }}=-48.3-66.6 \mu \mathrm{m}$; $\mathbf{R}_{\text {Laser }}=-33.4-66.7 \mu \mathrm{m}$. However, when comparing the LH and RH CMM arms or the axis of measurement, systematic differences were evident. In particular, in the $y$ - and $z$-axes, there was a greater error on a particular side when compared with the opposite horizontal arm. The worst-case systematic error between the two part coordinate frames for a particular E-Cube surface point was $42.1 \mu \mathrm{m}$.

Table 2 provides the median, minimum and maximum $2 \sigma$ random error values by axis for both the $\mathrm{LH}$ and $\mathrm{RH}$ horizontal arms. The repeatability of the touch trigger probe was

Table 1 Systematic error between the touch trigger and laser scanning probe for the E-Cube surface point measurements-median value (minimum value, maximum value) systematic errors per axis for each CMM horizontal measurement arm

\begin{tabular}{llccc}
\hline & & $\begin{array}{l}\text { Touch trigger probe/laser } \\
\text { scanning probe }-\mathbf{R}_{\text {Touch }}(\mu \mathrm{m})\end{array}$ & $\begin{array}{l}\text { Touch trigger probe/laser } \\
\text { scanning probe }-\mathbf{R}_{\text {Laser }}(\mu \mathrm{m})\end{array}$ & $\begin{array}{l}\text { Difference } \\
(\mu \mathrm{m})\end{array}$ \\
\hline$X$-axis & LH & $31.0(\min :-48.3, \max : 42.2)$ & $7.2(\min :-6.6, \max : 16.0)$ & $23.8(\min : 18.1, \max : 26.6)$ \\
& RH & $13.7(\min :-3.9, \max : 15.3)$ & $-6.9(\min :-24.3, \max :-3.7)$ & $20.4(\min : 19.1, \max : 20.6)$ \\
$Y$-axis & LH & $63.6(\min : 48.2, \max : 66.6)$ & $61.2(\min : 33.9, \max : 66.7)$ & $2.6(\min :-0.2, \max : 14.3)$ \\
& RH & $-33.2(\min :-41.8, \max :-26.8)$ & $-4.2(\min :-33.4, \max : 4.9)$ & $-29.0(\min :-31.7, \max :-8.4)$ \\
$Z$-axis & LH & $46.7(\min :-44.1, \max : 49.8)$ & $57.4(\min :-2.1, \max : 65.5)$ & $-18.8(\min :-42.1, \max :-7.7)$ \\
& RH & $23.0(\min :-5.0, \max : 35.8)$ & $27.1(\min :-1.0 . \max : 33.1)$ & $-4.0(\min :-4.1, \max : 4.6)$ \\
\hline
\end{tabular}


Table 2 Random error of the touch trigger probe compared to the laser scanner for the E-Cube surface point measurements-median value (minimum value, maximum value) systematic errors per axis for each CMM horizontal measurement arm

\begin{tabular}{lllcc}
\hline & & $\begin{array}{l}\text { Touch trigger probe } \\
(\mu \mathrm{m})\end{array}$ & $\begin{array}{l}\text { Laser scanning probe }-\mathbf{R}_{\text {Touch }} \\
(\mu \mathrm{m})\end{array}$ & $\begin{array}{l}\text { Laser scanning probe - } \\
(\mu \mathrm{m})\end{array}$ \\
\hline$X$-axis & LH & $4.9(\min : 2.9, \max : 5.3)$ & $11.5(\min : 10.1, \max : 15.8)$ & $10.7(\min : 3.4, \max : 19.1)$ \\
& RH & $5.1(\min : 3.9, \max : 5.4)$ & $7.5(\min : 3.1, \max : 8.7)$ & $7.2(\min : 3.8, \max : 9.4)$ \\
$Y$-axis & LH & $7.0(\min : 5.7, \max : 7.8)$ & $15.9(\min : 15.2, \max : 17.3)$ & $21.2(\min : 13.5, \max : 21.4)$ \\
& RH & $8.9(\min : 8.4, \max : 11.9)$ & $7.1(\min : 4.9, \max : 8.8)$ & $15.2(\min : 9.2, \max : 16.8)$ \\
$Z$-axis & LH & $7.3(\min : 3.3, \max : 8.1)$ & $14.5(\min : 10.6, \max : 20.1)$ & $20.6(\min : 8.7, \max : 30.2)$ \\
& RH & $3.0(\min : 2.9, \max : 4.0)$ & $4.3(\min : 4.1, \max : 5.6)$ & $9.8(\min : 8.4, \max : 11.0)$ \\
\hline
\end{tabular}

better in all cases except for RH E-Cube surface point measurements taken in the $y$-axis by the laser scanner using the $\mathbf{R}_{\text {Touch }}$ local coordinate frame. Repeatability was better with laser scanner surface point measurements taken with the RH horizontal CMM arm compared with the LH CMM arm. Comparing laser scanner measurements, repeatability was better for 12 of the $18 \mathrm{E}$-Cube surface points when using the $\mathbf{R}_{\text {Touch }}$ coordinate frame as opposed to the $\mathbf{R}_{\text {Laser }}$ coordinate frame.

The error between the local coordinate frames when constructed using measurements of the E-Cube datum plates with the touch trigger probe $\left(\mathbf{R}_{\text {Touch }}\right)$ compared to the laser scanner $\left(\mathbf{R}_{\text {Laser }}\right)$ showed differences in both their position and orientation, as Fig. 5 shows. There was a translational difference between the coordinate frame origins of $21.6 \mu \mathrm{m}$ $(2 \sigma=6.7 \mu \mathrm{m})$ in the $x$-axis, $4.7 \mu \mathrm{m}(2 \sigma=5.9 \mu \mathrm{m})$ in the $y$-axis and $4.1 \mu \mathrm{m}(2 \sigma=17.6 \mu \mathrm{m})$ in the $z$-axis. There was also an angular difference between the axes of $0.3^{\circ} \times 10^{-3}\left(2 \sigma=0.3^{\circ} \times 10^{-3}\right)$ for the $x$-axis, $1.4^{\circ} \times$ $10^{-3}\left(2 \sigma=1.1^{\circ} \times 10^{-3}\right)$ for the $y$-axis and $1.4^{\circ} \times 10^{-3}$ $\left(2 \sigma=1.0^{\circ} \times 10^{-3}\right)$ for the $z$-axis.

The effect of coordinate frame variation between $\mathbf{R}_{\text {Touch }}$ and $\mathbf{R}_{\text {Laser }}$ upon positional error is shown in Fig. 6. The mean positional error in the $x$-axis was $20.6 \mu \mathrm{m}$
$(2 \sigma=9.5 \mu \mathrm{m})$, compared to $-27.0 \mu \mathrm{m}(2 \sigma=40.2 \mu \mathrm{m})$ in the $y$-axis and $-16.4 \mu \mathrm{m}(2 \sigma=45.1 \mu \mathrm{m})$ in the $z$-axis. Figure 6 shows that the positional error in the $x$-axis is constant across all surface points. However, the positional error in the $y$ - and $z$-axes is dependent upon the location of the surface point. In the $y$-axis, the positional error is perfectly correlated $(r=1)$ between respective surface points on the LH and RH sides of the E-Cube. For the $z$-axis, once the effect of the $-16.4 \mu \mathrm{m}$ zero error is removed, the positional error for LH surface points are perfectly inversely correlated $(r=-1)$ with their respective RH surface points.

Table 3 presents the results of the crossed two-factor ANOVA test comparing the laser scanner measurement results of the E-Cube surface points using the $\mathbf{R}_{\text {Touch }}$ and $\mathbf{R}_{\text {Laser }}$ coordinate frames. The coordinate frame used did not have an effect upon the measurement error of the laser scanner compared to the touch trigger probe, $F(1,17)=0.002$, $p=0.967$. The mean error of the laser scanning probe compared to the touch trigger probe was therefore $-12.8 \mu \mathrm{m}$ $(2 \sigma=7.4 \mu \mathrm{m})$. The location of an individual surface point within three-dimensional space had a significant effect upon measurement agreement of the laser scanner compared to the touch trigger probe, $F(17,17)=12.308, p<0.001$, having a large overall effect, $\omega^{2}=0.925$. Figure 7 plots the
Fig. 5 Systematic error in position and orientation of local part coordinate frame when constructed using measurements from the laser scanner compared to the touch trigger probe

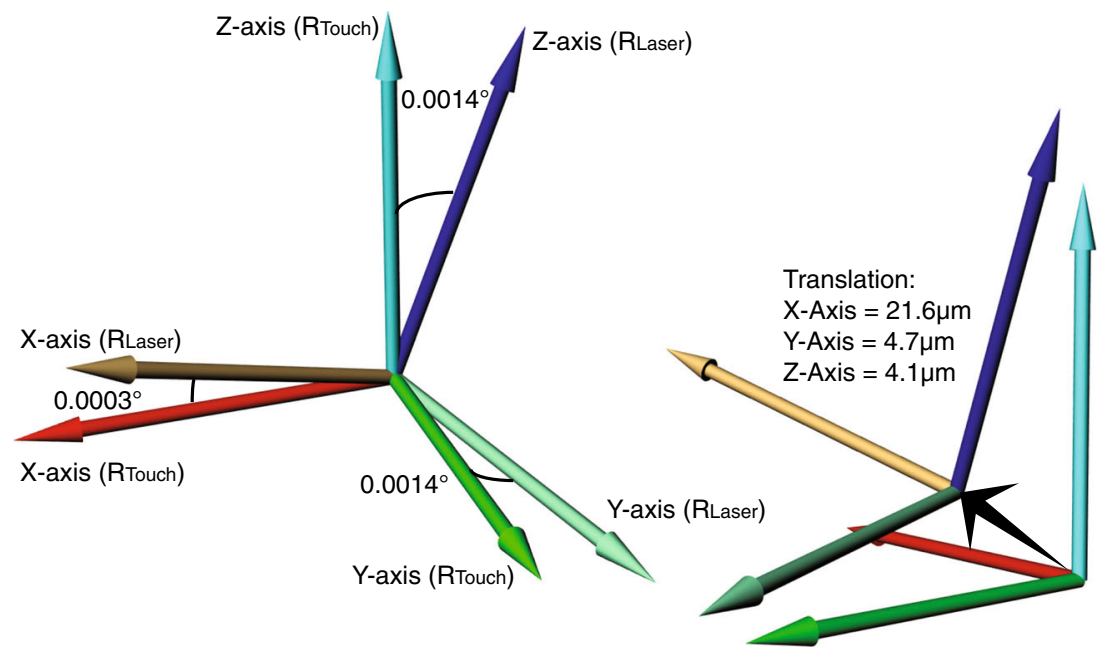




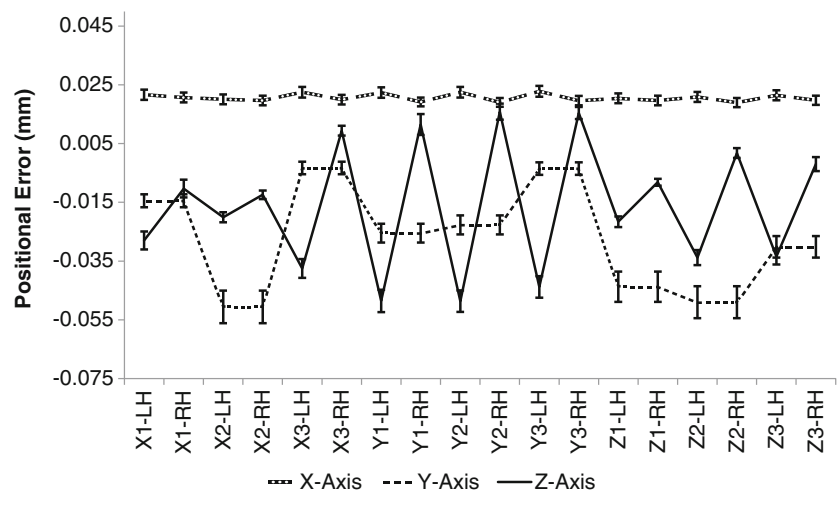

Fig. 6 Positional error between $\mathbf{R}_{\text {Touch }}$ and $\mathbf{R}_{\text {Laser }}$ local part coordinate frames for each E-Cube surface point (error bar $95 \%$ confidence interval)

average deviation per E-Cube surface point and shows that systematic error was less for the RH horizontal CMM arm compared to the LH CMM arm. There was also a significant interaction between the location of the E-Cube surface point and the coordinate frame used to measure that surface point in relation to the systematic error with touch trigger probe measurements, $F(17,1,044)=137.483, p<0.001$, having a medium overall effect, $\omega^{2}=0.075$. Figure 8 shows that for some surface points, laser scanner measurement using the $\mathbf{R}_{\text {Laser }}$ coordinate frame had better measurement agreement than the equivalent laser scanner measurements using the $\mathbf{R}_{\text {Touch }}$ coordinate frame. However, there were cases where the systematic error increased, while there were other surface point measurements where the coordinate frame used did not affect measurement agreement with the touch trigger probe.

\section{Discussion}

The measurement of components and assemblies using a CMM fitted with a touch trigger probe is considered to be the most reliable and accurate method of quality control in many industries [2]. This technology uses discrete surface

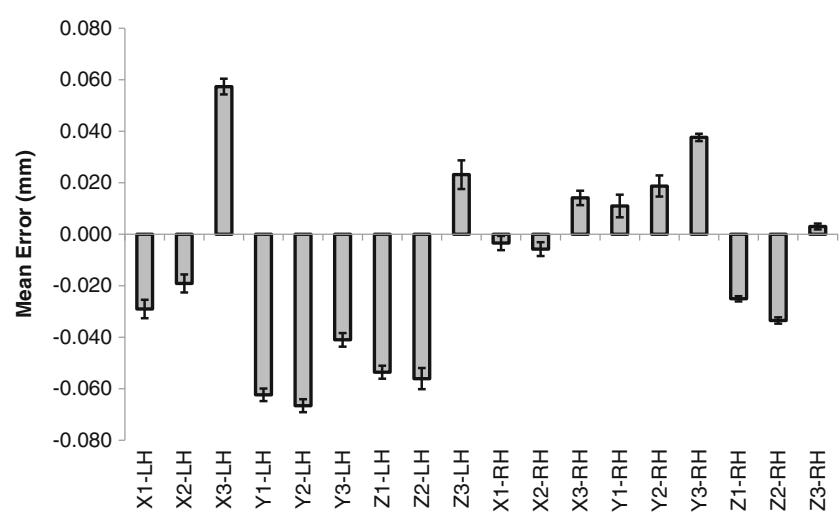

Fig. 7 Laser scanner systematic error compared to reference touch trigger probe for each E-Cube surface point measurement (error bar $95 \%$ confidence interval)

point measurements to construct ideal geometric elements such as circles and cylinders to compare with nominal part geometry in order to identify errors in the production process [28]. However, there is a trade-off between measurement time and the amount of individual surface points which can be measured [4]. This can lead to badly conditioned fitting algorithms as well as a less than ideal amount of measurement data to be able to inform about the production process characteristics $[7,13]$. Laser scanning on the other hand is considered less accurate but it is able to collect a much greater density of measurement points within the same measurement time [4]. This can potentially solve the problems described with touch trigger probes only if the measurement accuracy is acceptable for the specific application. However, it is important that any potential advantages are evaluated within the context of its intended measurement task. This is due to complex CMM measurements involve providing information such as three-dimensional locations, profiles and angles in regions of the measurement volume that may not have been evaluated during CMM verification [22]. Consequently, CMM performance as evaluated through the measurement of a calibrated test length should be considered the baseline from which to begin any further CMM evaluation.

Table 3 Crossed two-factor ANOVA comparing the effect of constructed coordinate frame and the spatial location of E-Cube surface point upon measurement agreement of the laser scanner with the touch trigger probe

\begin{tabular}{llcrr}
\hline & Sum of squares & Degrees of freedom & Mean square & $F$ value \\
\hline Coordinate frame & 0.00001 & 1 & 0.00001 & 0.002 \\
Location of surface point & 1.31925 & 17 & 0.07760 & 0.967 \\
Coordinate frame * surface point & 0.10719 & 17 & 0.00631 & 12.308 \\
Error & 0.04788 & 1044 & 0.00005 & $<0.001$ \\
Total & 1.47433 & 1079 & & $<0.001$ \\
\hline
\end{tabular}




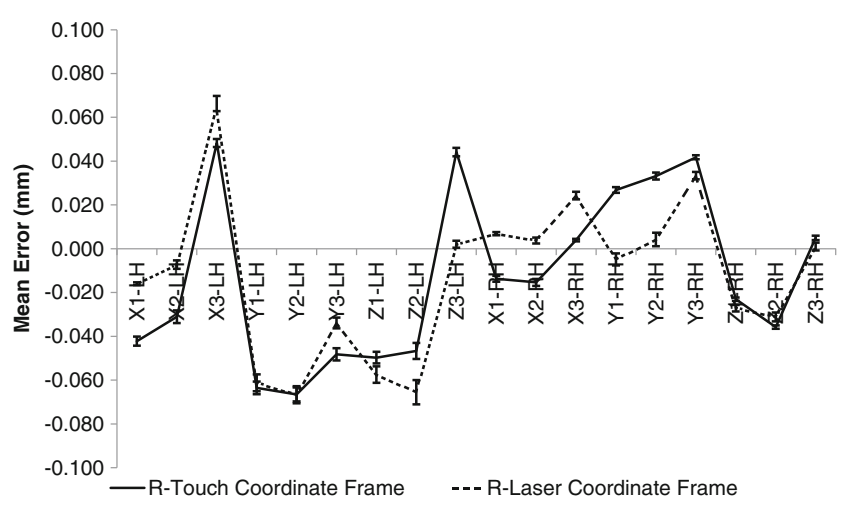

Fig. 8 Systematic error by E-Cube surface point between laser scanner measurements made with $\mathbf{R}_{\text {Touch }}$ and $\mathbf{R}_{\text {Laser }}$ local part coordinate frames (error bar $95 \%$ confidence interval)

\subsection{Assessment of CMM}

There have been a number of studies which have evaluated the performance of a laser scanner against a reference touch trigger probe using a reference sphere [5-7]. When using a sphere, problems occur with measurement agreement between the probing technologies in the estimation of the $z$-axis sphere centre position and sphere diameter [17]. This is due to only a segment of sphere being measured with the laser scanner, the effect of which can be reduced by increasing the reference sphere diameter [5]. The sphere used in this study was a similar size to the one used by Isheil et al. [7] and the error in diameter estimation using the laser scanning probe of between 22.8 and $27.1 \mu \mathrm{m}$ was within the range reported by this study. Furthermore, deviation in $z-$ axis compared to the touch trigger probe was comparable to the results reported by Martinez et al. [5]. Consequently, the performance of the measurement system used in this study is similar to those used in previous studies.

In the automotive industry, to be able to get access to all sections of the vehicle, a dual horizontal arm CMM configuration is very often employed. Consequently, different CMM arms measure the $\mathrm{LH}$ and $\mathrm{RH}$ sides of the vehicle using a common part coordinate frame. This has an impact with regard to measurement uncertainty, as any error between the two CMM horizontal arms will affect the spatial relationship between the measured datum features. Consequently, this will influence both the position and orientation of the constructed local part coordinate frame. When using a touch trigger probe, a maximum systematic error between the LH and RH horizontal CMM arms was found to be $11.0 \mu \mathrm{m}$, changing to a laser scanner increased this systematic error to $19.6 \mu \mathrm{m}$. Therefore, the construction of a local part coordinate frame using dual arm CMM measurements made with the laser scanner would be affected to a greater degree compared to the same measurements made with the touch trigger probe.

\subsection{Effect of local part coordinate frame}

The deviation between the local part coordinate frames constructed from measurements using the laser scanner compared to touch trigger probe measurements was evaluated in this study. The results show that there were differences in both the position of the respective coordinate frames and in their orientation. This was affected by the datum precedence specified in the experimental set-up, with three $z$-points in contact with the primary plane, two $y$-points in contact with the secondary plane and one $x$-point in contact with the tertiary plane. This datum precedence meant that the $y$-and $z$-axes were primarily affected by the orientation between the axes of the two local part coordinate frames, as Fig. 5 illustrates. In contrast, the $x$-axis was primarily affected by a translational error between the local part coordinate frame origins. This affected the positional error in each axis between the $\mathbf{R}_{\text {Touch }}$ and $\mathbf{R}_{\text {Laser }}$ coordinate frames, as Fig. 6 shows. The $x$-axis positional error was constant for all E-Cube surface point locations, while positional error in the $y$ - and $z$-axes was dependent upon the location of the surface point within the local part coordinate frame.

The effect that the positional error between the $\mathbf{R}_{\text {Touch }}$ and $\mathbf{R}_{\text {Laser }}$ local part coordinate frames have upon measurement error was evaluated using a crossed two-way ANOVA. The results showed that the local coordinate frame used, while not significant in itself, did have a significant effect upon measurement agreement with the touch trigger probe depending upon the particular location of a E-Cube surface point in three-dimensional space, as Fig. 8 shows. For the surface points measured in the $x$-axis, the agreement was independent of location with a $20.6 \mu \mathrm{m}$ offset between $\mathbf{R}_{\text {Touch }}$ laser scanner measurements and $\mathbf{R}_{\text {Laser }}$ measurements. For the $y$ - and $z$-axes, laser scanner measurement agreement with the reference touch trigger probe improved for some E-Cube surface points when using the $\mathbf{R}_{\text {Laser }}$ local part coordinate frame, but for other surface points agreement either deteriorated or remained unaffected by the coordinate frame used.

\subsection{Effect of surface point location}

The overall effect size that surface point positional error between the local part coordinate frames had upon laser scanner measurement agreement, as measured by the $\omega^{2}$ test-statistic, was within the medium range defined by Field [27]. The ANOVA test revealed a much larger effect upon laser scanner measurement agreement with the touch trigger probe was caused by the location of the E-Cube surface point itself, independent of the local coordinate frame used, as Fig. 7 shows. This implies that other factors, such as probe head orientation or incident angle, have a greater 
effect upon measurement agreement compared to which probing technology was used to measure the datum features from which to construct the local part coordinate frame. The most notable difference in Fig. 7 is the lower systematic error of laser scanner measurements taken with the RH CMM horizontal arm compared to the LH CMM arm. The mean systematic error for the LH E-Cube surface points was $45.4 \mu \mathrm{m}$ compared to $16.9 \mu \mathrm{m}$ for the RH surface points. The only notable difference between the two sides of the E-Cube was the three emergency lights located on the LH wall of the laboratory. Cuesta et al. [12] stated that the halogen light spectrum and laser light spectrum overlap. This means that when a laboratory is not lit uniformly, the percentage of energy in the laser scanner image that does not come from the laser light emission will be influenced by the location of the measurement. Consequently, this affects the laser scanner accuracy both across the measurement scale and between horizontal CMM arms. Therefore, further work is required to assess whether lighting conditions affect measurement uncertainty using the latest generation of laser scanning systems.

It has been stated that CMM mounted laser scanners are at least one order of magnitude less accurate than touch trigger probe [6]. Although, more recent studies have found an improvement is laser scanner accuracy [3, 5], which this study has also found. Considering measurements against a known diameter, the touch trigger probe measurement results deviated by at worst $16.7 \mu \mathrm{m}$ compared to $27.1 \mu \mathrm{m}$ when the sphere diameter was measured with the laser scanner. This $10.4 \mu \mathrm{m}$ difference is further supported by the $12.8 \mu \mathrm{m}$ mean measurement error between the probing technologies as found by the twoway crossed ANOVA. However, the difference in agreement does not act uniformly when assessed over the complete coordinate frame of the vehicle. Instead, the measurement deviation between the probing technologies was found to be as low as $1.0 \mu \mathrm{m}$ for some surface points but as a high as $66.7 \mu \mathrm{m}$ in the worst case. This effect is also true for random error, with the touch trigger probe having a mean $2 \sigma$ repeatability of $5.9 \mu \mathrm{m}$ and a range of $2.9-11.9 \mu \mathrm{m}$. In comparison, the laser scanner had a mean $2 \sigma$ repeatability of $10.3 \mu \mathrm{m}(3.1-20.1 \mu \mathrm{m})$ with the $\mathbf{R}_{\text {Touch }}$ local coordinate frame and a mean $2 \sigma$ repeatability of $13.3 \mu \mathrm{m}$ (3.4-30.2 $\mu \mathrm{m})$ with the $\mathbf{R}_{\text {Laser }}$ coordinate frame. Therefore, these results support the previous findings that laser scanners are not as accurate or repeatable as the touch trigger probe. However, if the repeatability values of the $\mathbf{R}_{\text {Touch }}$ laser scanner measurements are taken, the accuracy and repeatability difference with the touch trigger probe is no longer one order of magnitude but less than a factor of two.

\section{Conclusion}

This study had the objective of comparing the random and systematic error inherent in a horizontal dual arm CMM using a multi-sensor probing system in an automotive environment. To do this, a full scale automotive artefact with the same geometry and similar surface properties as a vehicle body was used. It has been found, taking the worst case, that there was a systematic error of $66.7 \mu \mathrm{m}$ between the touch trigger probe and the laser scanner. The worst case $\pm 2 \sigma$ repeatability using the $\mathbf{R}_{\text {Touch }}$ local part coordinate frame was $\pm 20.1 \mu \mathrm{m}$. Taking the 1:8 measurement uncertainty - tolerance interval ratio specified within the geometrical product specifications (GPS), a tolerance $\pm 0.5 \mathrm{~mm}$ can be measured with confidence using a multisensor system [10]. In addition, the performance of laser scanners are now approaching the standard of touch trigger probes. Consequently, the two technologies can be used together as part of a multi-sensor data fusion measurement system to provide improved quality of the measurement result, as well as reliable evaluation of features which may not be accessible with single sensor systems [11]. However, constructing a local part coordinate frame using laser scanner measurements has a negative effect upon measurement repeatability and makes measurement agreement with the touch trigger probe less predictable. Furthermore, measurement agreement between probing technologies is dependent upon the location of the surface feature. This has implications with regard to conducting the automotive gauge repeatability and reproducibility evaluation. The reason for this is that every feature is required to be measured, rather than just a subset, because certain feature locations may have a better agreement and repeatability than others. If these factors are considered, then a multi-sensor CMM system can be adopted in an automotive environment. Where the speed advantages of the laser scanner is used for features which have good agreement and repeatability, while the accuracy of the touch trigger probe is used for those features that cannot be measured reliably with the laser scanner. Further work needs to be done to evaluate the multisensor approach with the measurement of other surface features such as circular holes, round slots and cylindrical pins.

Acknowledgements This work was carried out as part of the Product Evaluation Technologies Group, part funded by the High Value Manufacturing Catapult. The LK H Horizontal Dual Arm CMM was part funded by Advantage West Midlands and the European Regional Development Fund. The authors would also like to acknowledge the support of Paul Peterson at Jaguar Land Rover. 
Open Access This article is distributed under the terms of the Creative Commons Attribution License which permits any use, distribution, and reproduction in any medium, provided the original author(s) and the source are credited.

\section{References}

1. Zhao H, Kruth J-P, Van Gestel N, Boeckmans B, Bleys P (2012) Automated dimensional inspection planning using the combination of laser scanner and tactile probe. Measurement 45:10571066

2. Martínez S, Cuesta E, Barreiro J, Álvarez B (2010) Analysis of laser scanning and strategies for dimensional and geometrical control. Int J Adv Manuf Technol 46:621-629

3. Muralikrishnan B, Ren W, Stanfield E, Everett D, Zheng A, Doiron T (2013) Applications of profile filtering in the dimensional metrology of fuel cell plates. Meas Sci Technol 24. doi:10.1088/0957-0233/24/6/065003

4. Bešić I, Van Gestel N, Kruth J-P, Bleys P (2011) Accuracy improvements of laser line scanning for feature measurements on CMM. Opt Lasers Eng 49:1274-1280

5. Martínez S, Cuesta E, Barreiro J, Álvarez B (2010) Methodology for comparison of laser digitizing versus contact systems in dimensional control. Opt Lasers Eng 48:1238-1246

6. Feng H-Y, Liu Y, Xi F (2001) Analysis of digitizing errors of a laser scanning system. Precis Eng 25:185-191

7. Isheil A, Gonnet J-P, Joannic D, Fontaine J-F (2011) Systematic error correction of a 3D laser scanning measurement device. Opt Lasers Eng 49:16-24

8. Kiraci E, Attridge A, Williams MA (2012) The use of laser scanning technology to improve the design process. Appl Mech Mater 110:4188-4122

9. Abenhaim GN, Desrochers A, Tahan A (2012) Nonrigid parts' specification and inspection methods: notions, challenges, and recent advancements. Int J Adv Manuf Technol 63:741-752

10. Mahmud M, Joannic D, Roy M, Isheil A, Fontaine J-F (2011) 3D part inspection path planning of a laser scanner with control on uncertainty. Comput Aided Design 43:345-355

11. Weckenmann A, Jiang X, Sommer KD, Neuschaefer-Rube U, Seewig J, Shaw L, Estler T (2009) Multisensor data fusion in dimensional metrology. CIRP Ann-Manuf Techn 58:701-721

12. Cuesta E, Carlos Rico J, Fernández P, Blanco D, Valiño G (2009) Influence of roughness on surface scanning by means of a laser stripe. Int J Adv Manuf Technol 43:1157-1166

13. Shakarji CM (2012) Coordinate measurement system algorithms and filters. In: Hocken RJ, Pereira PH (eds) Coordinate measuring machines and systems, 2nd edn. CRC Press, Boca Raton, pp 153182
14. Weckenmann A, Estler T, Peggs G, McMurty D (2004) Probing systems in dimensional metrology. CIRP Ann-Manuf Techn $54: 657-684$

15. Contri A, Bourdet P, Lartigue C (2002) Quality of 3D digitised points obtained with non-contact optical sensors. CIRP Ann-Manuf Techn 51:443-446

16. Vukašinović N, Bračun D, Možina J (2010) The influence of incident angle, object colour and distance on CNC laser scanning. Int J Adv Manuf Technol 50:265-274

17. Van Gestel N, Cuypers S, Bleys P, Kruth J-P (2009) A performance evaluation test for laser line scanners on CMMs. Opt Lasers Eng 47:336-342

18. Muralikrishnan B, Ren W, Everett D, Stanfield E, Doiron T (2012) Performance evaluation experiments on a laser spot triangulation probe. Measurement 45:333-343

19. Vukašinović N, Bračun D, Možina J, Duhovnik (2012) A new method for defining the measurement-uncertainty model of CNC laser-triangulation scanner. Int J Adv Manuf Technol 58:10971104

20. Vezzetti E (2009) Computer aided inspection: design of customerorientated benchmark for noncontact 3D scanner evaluation. Int $\mathrm{J}$ Adv Manuf Technol 41:1140-1151

21. Vukašinović N, Možina J, Duhovnik (2012) Correlation between incident angle, measurement distance, object colour and the number of acquired points at CNC Laser Scanning. Stroj Vestn-J Mech E 58:23-28

22. British Standards Institution (2009) BS EN ISO 10360-2:2009Geometrical Product Specifications (GPS)-Acceptance and reverification tests for coordinate measuring machines (CMM)Part 2: CMMs used for measuring linear dimensions, London, BSI

23. Attridge A, Williams M, Tennant C (2005) Achieving craftsmanship targets across the UK automotive supply base, through the use of quality maturation tools and processes. In: Proceedings of the SAE 2005 world congress. Detroit

24. Attridge A, Williams MA, Tennant C (2007) The role of physical modelling in the design verification stage of the automotive NPI process in the premium sector. Int $\mathbf{J}$ Automot Tech Manag 7:3254

25. Turley GA, Williams MA, Tennant C (2007) Final vehicle product audit methodologies within the automotive industry. Int J Prod Qual Manag 2:1-22

26. Kirkup L, Frenkel RB (2006) An introduction to uncertainty in measurement using the GUM (Guide to the expression of uncertainty in measurement). Cambridge University Press, Cambridge

27. Field A (2009) Discovering statistics using SPSS, 3rd edn. Sage Publications, London

28. Pereira PH (2012) Cartesian coordinate measuring machines. In: Hocken RJ, Pereira PH (eds) Coordinate measuring machines and systems 2nd edn. CRC Press, Boca Raton, pp 5779 